\title{
Experimental and Numerical Comparison Q-Switched Fiber Laser Generation using Graphene as Saturable Absorber
}

\author{
Noor Azura Awang*, Nor Syuhada Aziz, Atiqah Nabieha Azmi, Fatin Shaqira Hadi and Zahariah Zakaria \\ Science Department, Faculty Science, Technology and Human Development, University Tun Hussein Onn Malaysia, Parit Raja, \\ 86400, Batu Pahat, Johor
}

\begin{abstract}
We demonstrated the comparison experimentally and numerically a compact Q-switched erbium-doped fiber (EDF) laser based on graphene as a saturable absorber (SA). By optically driven deposition of graphene on a fiber core, the SA is constructed and inserted into a diode-pumped EDF laser cavity. Lasing in CW region starts at $10 \mathrm{~mW}$, whereas stable self-starting Q-switching with a central wavelength of $1530 \mathrm{~nm}$ begins at $18 \mathrm{~mW}$. In this paper, at $35 \mathrm{~mW}$, the maximum pulse energy reaches at 2 $\mu J$ with pulse repetition rate of $1 \mathrm{MHz}$ and the narrowest pulse width is around $10 \mu \mathrm{s}$ is obtained. The stability of the pulse is verified from the radio-frequency (RF) spectrum with a measured signal-to-noise ratio (SNR) of $48 \mathrm{~dB}$. In this study, the design is compared with the simulation using the Optisystem software. The output power of the experimental study is also compared with the simulation to examine the performance.
\end{abstract}

\section{Introduction}

Q-switched fiber laser based on saturable absorber has been useful in light source and widely potential for use in application such as telecommunication, medicine, fiber optic sensing, remote sensing, range finding and industrial processing [1]. The generation of short pulses is also called a Q-switching operation. A laser ring cavity requires a gain medium such as erbium-doped fiber (EDF) and also a 'Q-switcher' component in order to obtain stable short pulses. The most widely utilized Qswitching technologies employ a nonlinear effect, such as nonlinear polarization rotation (NPR) as reported in [2] or a nonlinear optical loop mirror (NOLM) as proposed in [3, 4], along with the use of a fast saturable absorber (SA) element such as semiconductor saturable absorber mirrors (SESAMs) [5], carbon nanotubes (CNT) [6] or graphene [7]. Chronology, first SESAM usually has drawbacks of cost-ineffective and timeconsuming fabrication process lastly has limited pulse [8] compare to CNT which is simple and cost effective alternative [9]. Graphene said by Pi Li Huang et al good in optically visualized, high transperancy and linear absorption that can be characterized in optical properties. Graphene as a novel nanomaterial can be used as a fast SA with wide spectrum range [7] few-layered graphene is needed for the implementation as SA.

There are few complicated fabrication methods of few-layered graphene, such as chemical-vapor deposition (CVD) [10], the laser-induced surface decomposition method, pulsed-laser deposition (PLD), chemical synthesis and the mechanical exfoliation [11] however we filter out the easier alternatives with comparable saturable-absorption performance. Graphene is still the most favourite selection in most experiment recently in mode-locked and Q-switched fiber laser.

Fiber that doped with rare earth element mostly used in research as active gain medium [12][13] such as Ytterbium (Yb) doped (YDFL), Erbium Doped (EDFL), $\mathrm{Er}$ and $\mathrm{Yb}$ co-doped (EYDFL), Bismuth (Bi) doped (BDFL), Praseodymium (Pr) doped (PDFL) , and Thulium (Th) doped (TDFL) [14].

In this work, we determined successful operation spectrum at $1530 \mathrm{~nm}$ of a Q-switched fiber laser. It only required a simple and cheap graphene SA. The graphene layer was fabricated using mechanical exfoliation method. This fiber laser is cooperation with two meters of erbium doped fiber (EDF) as a gain medium to amplified light when energy is pumped into active laser medium. The schematic setup between Q-switched and mode locked exactly the same. Q-switched fiber lasers ring are area of most interest because they have clear technical advantages. In a single ring, we use graphene as the SA because the features are almost ideal SA as fast recovery times, low saturation intensity, low cost and easy fabrication. The Q-switched fiber laser generation is compared with Optisystem software.

\footnotetext{
* Corresponding author: norazura@uthm.edu.my
} 


\section{Deposition and characterization grapheme on ferrule}

The first stage in setting up Q-switched fiber laser is deposited graphene on fiber ferrule as SA which plays role as a passive Q-switched. The process deposition of graphene was done using mechanical exfoliation technique. First, flakes of graphite were pasted onto a strip of scotch tape and pressed and peeled off repeatedly, in order to enhance the graphene thickness. The result of graphene strip was then pressed against the end face of the optical fiber ferrule. The scotch tape was slowly peeled off so that the graphene layer remained on the end facet of the ferrule and was connected to another fiber pigtail via a connector (FC/PC), to form a simple SA device for the fiber laser as shown in Fig. 1.
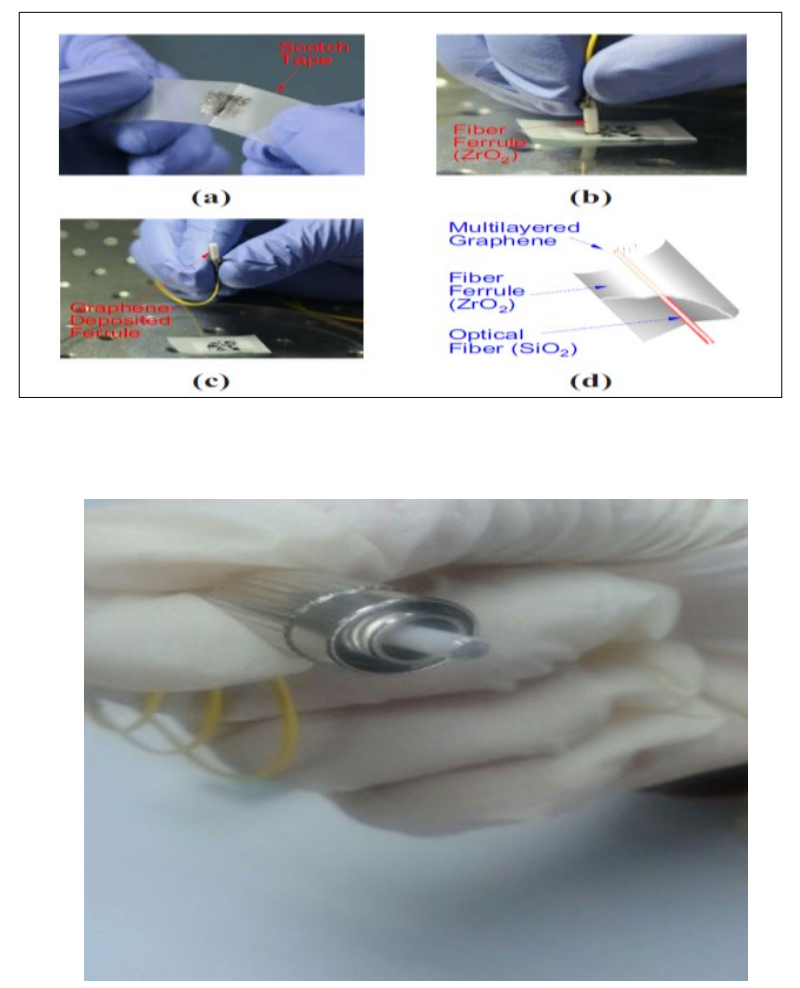

Fig. 1. Graphene deposited on ferrule by mechanical exfoliation

Fig. 2 shows (a) Single Natural Graphite flakes are opaque and have a metallic look under Meiji Microscope. After several times peels off, graphite flakes are identified under Optical Microscope as shown in Fig. 2(b). In Fig. 2(c) mechanical exfoliation processtransferred the graphite layers to the surface of the fiber ferrule identifying by fiberscope. Fig. 2(d) Scanning electron microscopy (SEM) of the optical fibers with graphite flakes optical deposited at core region.

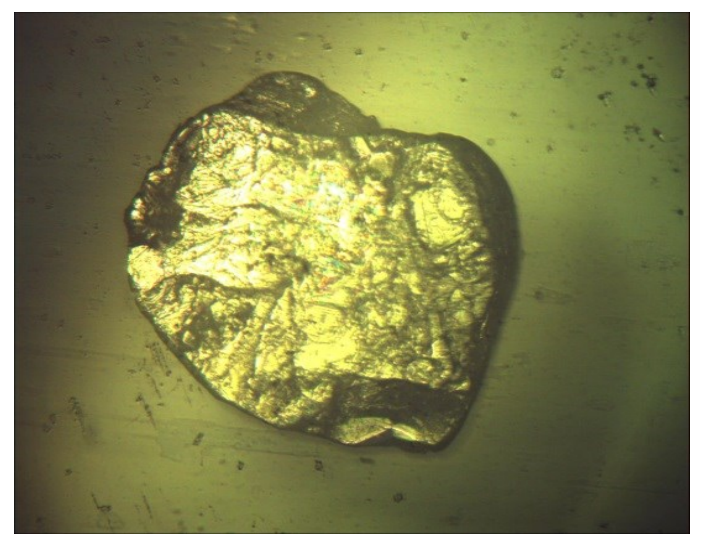

(a)

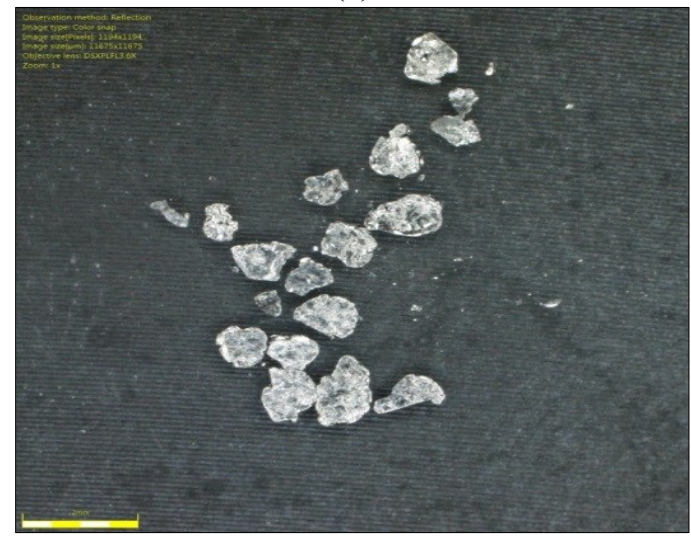

(b)

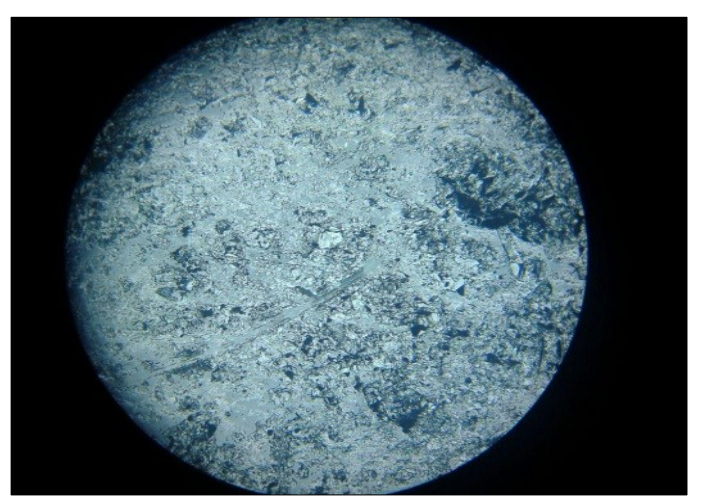

(c)

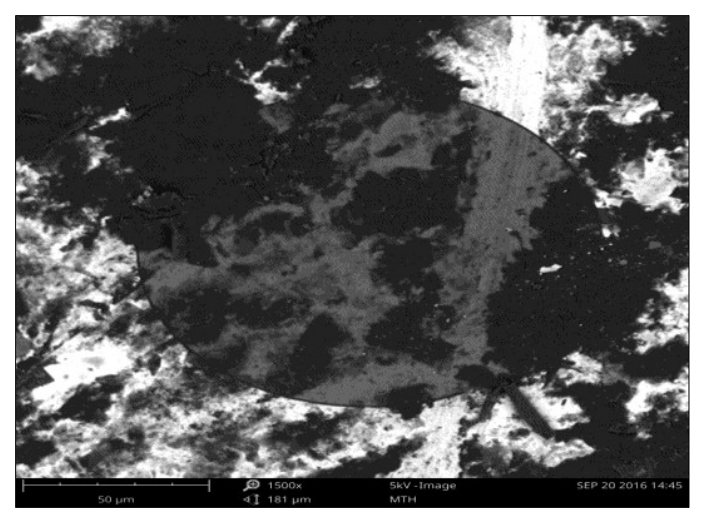

(d)

Fig. 2. Graphene under a) Meiji Microscope, b) Optical Microscope, c) Fiberscope, d) Scanning electron microscopy (SEM) 
The graphene layers were transferred onto the fiber ferrule, a series of measurements including scanning electron microscopy (SEM) and energy dispersive X-ray spectroscopy (EDS) were conducted so that entirely cover of fiber core clearly seen. Fig. 3 shows the images of graphene on ferrule by mechanical exfoliation method under Semiconductor Electron Microscope (SEM). The image was zoomed on the core surface of the ferrule. Graphite flakes on ferrule close up using scanning electron microscope. SEM image micrograph of graphene ferrule on the core arrange loosely around the core. Dark grey spot on fiber core consist of high content carbon while light grey spot content low of carbon.

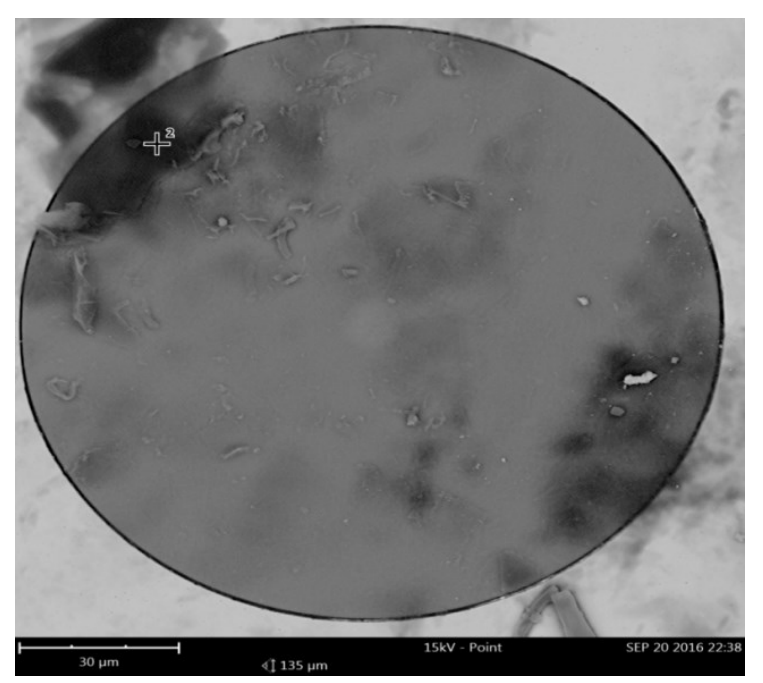

Fig. 3. Fiber-end with Graphene optically-deposited in the core area

Fig. 4 shows the EDS analysis on the ferrule deposited with graphene. The analysis of the percentage of element was placed on Table 1. The EDS have traced three elements on fiber ferrule such as silicon, carbon and zirconium. The EDS observed the silicon existed because the fiber ferrule is made of silicon which represented by the surface part of the ferrule.

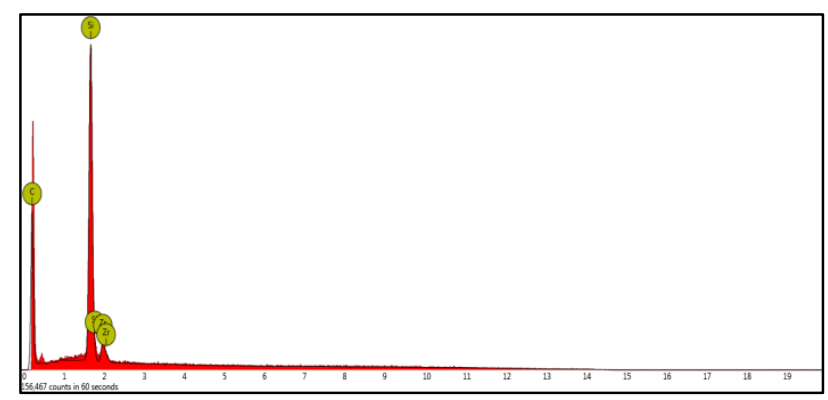

Fig. 4. Energy Dispersive Spectrum (EDS) of thegraphite flakes deposited onfiber ferrule.
Table 1. Percentage of elements on ferrule deposited with graphite.

\begin{tabular}{|c|c|c|}
\hline Element & Weight (\%) & Atomic (\%) \\
\hline Silicon & 16.44 & 8.10 \\
Carbon & 79.14 & 91.22 \\
Zirconium & 4.42 & 0.67 \\
\hline
\end{tabular}

The composition of the transferred graphene was confirmed by the energy dispersive spectroscopy (EDS). EDS is a technique that analyzes X-rays generated by the bombardment of the sample by an electron beam. The analysis of the spectroscopy data presented in Fig. 4 above confirms that the transferred material have a strong intensity peak of Carbon, along with small portions of Silicon and Zirconium that existed because of the fiber ferrules that were used. The silicon originate from the substrate ferrule which is the optical fiber made from high purity silicone dioxide ( $\mathrm{SiO} 2)$. Graphene has natural form of hexagonal crystal structure and consist of pure carbon. Based on study, chemicalsymbol of graphite is $\mathrm{C}$ referring to most well-known allotropes of carbon,which purely consists of carbon. It is proved under EDS the carbon existed on ferrule.

\section{Experimental setup}

Fig. 5 shows a design of Q-switched fiber laser. It includes a $980 \mathrm{~nm}$ laser diode as a pump source, a wavelength division multiplexer (WDM), a two meter long EDF as a gain medium, an optical isolator, polarization controller (PC), graphene SA prepared above, 90:10 dB output coupler, 50:50 output coupler, oscilloscope (OSC) and optical spectrum analyser (OSA). It was pumped by a $980 \mathrm{~nm}$ diode laser via a wavelength division multiplexer (WDM). An isolator was employed to ensure the unidirectional operation, a polarization controller employed to fine tune the linear cavity birefringence and utilized to adjust the polarization for Q-switched optimization. The light will transmit to the 90:10 directional optical coupler which $10 \%$ maintain as an outputand the another $90 \%$ will be continuously transmitted to the fiber ring. The $10 \%$ optical coupler connected to the 50:50 optical coupler which split the $10 \%$ of the output power to OSC and OSA by $50 \%$ respectively. The propagation processes would occur inside the coupler, so the output spectrum spectral resolution $20.0 \mathrm{~nm} / \mathrm{div}$ and repetition rate were shown by OSA and OSC.

Experiments of Q-switched fiber laser can be compare between optical system software. Optisystem is the optical communication system design software that enables users to plan, test, and simulates optical links in the transmission layer of modern optical networks. This software can create a model of an optical system, execute the model and view measures of the system's performance. The optical system demonstrated a fiber laser, based on the passive Q-switched using SA as shown in Fig. 6. It is a unidirectional ring cavity and every component in the software application acted as a real component in the fiber laser. 


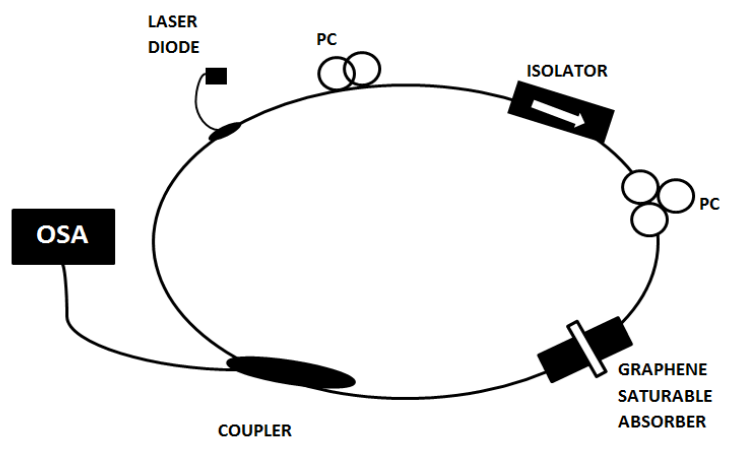

Fig. 5. Schematic configuration of single Q-switch fiber laser.

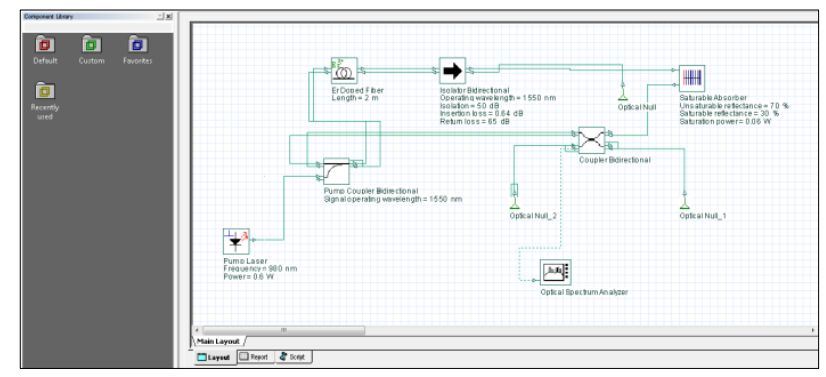

Fig. 6. Schematic circuit simulation for single ring in optisystem

\section{Results and Discussion}

In experimental, broad wavelength can be generated in the range of $1500 \mathrm{~nm}$ until $1600 \mathrm{~nm}$ in C-band and Qswitched is available this spectrum range which trends in this research. The Fig. 7 the optical spectrum taken from the OSA at central wavelength of $1550 \mathrm{~nm}$. Lowest pump current $50 \mathrm{~mA}$ did not appear any single peak wavelength. Q-switched start to appear at current 300 $\mathrm{mA}$ and consistency increasing the peak with increasing the current value. The Q-switching operation starts at pump current $300 \mathrm{~mA}$ and continues until $600 \mathrm{~mA}$.

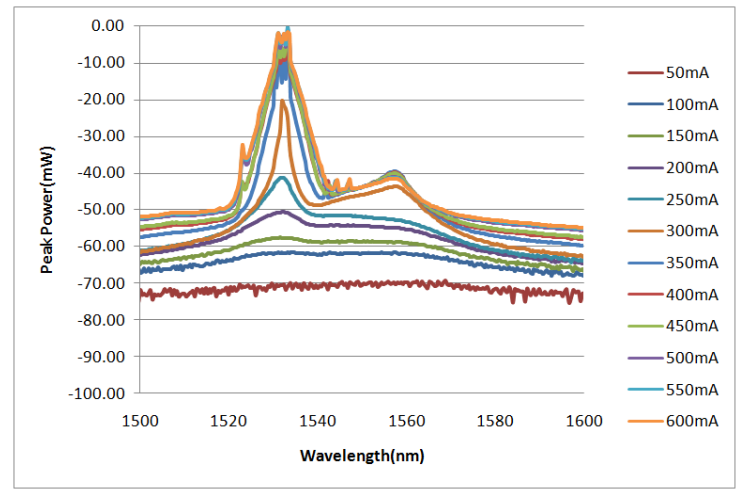

Fig. 7. Output spectra of the Q-Switch at different current pump power.
The spectrum seemed to have little change when increased the pump current and show a good Q-switched stability as shown in Fig. 7. The spectral bandwidthis $13.6 \mathrm{~nm}$ at $600 \mathrm{~mA}$ was centered at $1530 \mathrm{~nm}$, measured by an optical spectral analyzer with resolution of $20 \mathrm{~nm} /$ div. The maximum peak power was $2.53 \times 10^{-6}$ $\mathrm{mW}$ at $600 \mathrm{~mA}$ pump current.

In experimental, stable Q-switched starts when pump power reach by slightly tuning the PC. The fiber laser can operate in a stable Q-switched state at optical spectrum as shown in Fig. 8. This output spectrum was obtained by using optical spectrum analyser (MS9740A). Q-switched works at the wavelength of $1530 \mathrm{~nm}$ with output power is generate spectral bandwidth $13.6 \mathrm{~nm}$. For comparison purpose with simulation, the spectrum was combined in a single graph as shown in Fig. 4. Both of the experimental result and simulation result of Qswitched fiber laser was compared.

The optical system which using simulation demonstrates a fiber laser, based on passive Q-switched a SA, which efficient in producing short pulse, however due to limitation of study we study the bandwidth between experiment and simulation as shown in Fig. 8 . By comparing, the spectral bandwidth of the simulation is $9 \times 10^{-9} \mathrm{~nm}$. A slight different value between this two bandwidth, can be said due to loss in fiber during progress experiment. The experimental result is more efficient compare to simulation because experimental exceed high power, which is needed the most to carry information for long haul - transmission.

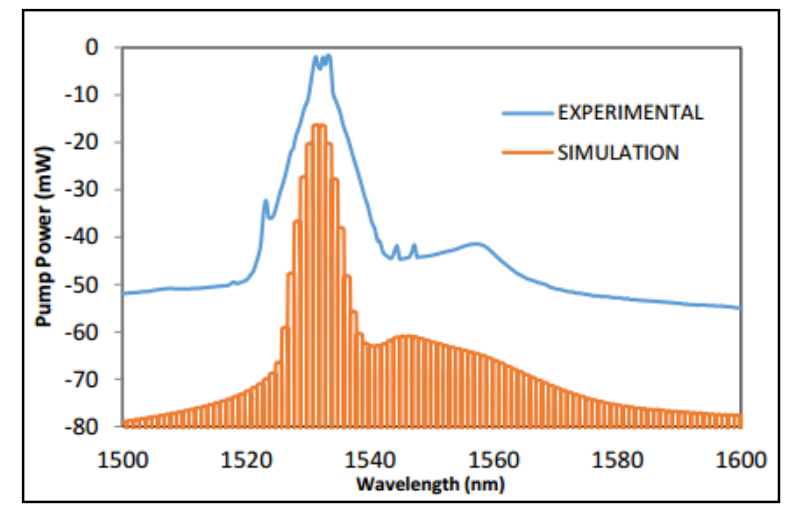

Fig. 8. Result comparison between experimental and simulation.

The definition of a threshold is the entrance or start of something. In Fig. 9 threshold cut level wavelength is $3.00 \mathrm{~dB}$. This is to identify spectrum width $(\Delta \lambda)$ for each pump power after q-switched started to appear. The spectrum width minor fluctuated between $2.00-3.00 \mathrm{~nm}$ started from pump current $350 \mathrm{~mA}$ which pump power $17.25 \mathrm{~mW}$, threshold product $1.179 \mathrm{~nm}$. Stable threshold can be seen during pump current $350 \mathrm{~mA}$ until $550 \mathrm{~mA}$. 


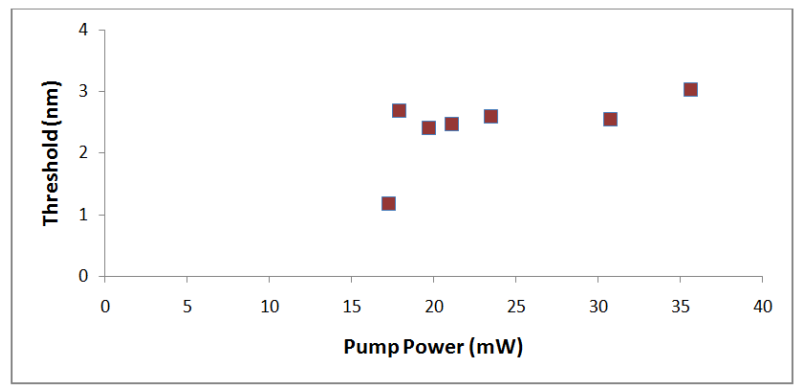

Fig. 9. Threshold against Pump Power

A stable Q-switched is self-started when pump power is fixed at pump power. The measured OSA were recorded every $5 \min$ for $1 \mathrm{~h}$ as shown in Fig. 10. A stable operation is clearly observed for Q-Switched. It can be seen that the overall shape Q-switched spectra remain unchanged despite a slight time shifts and optical power drift due to environmental temperature changes.

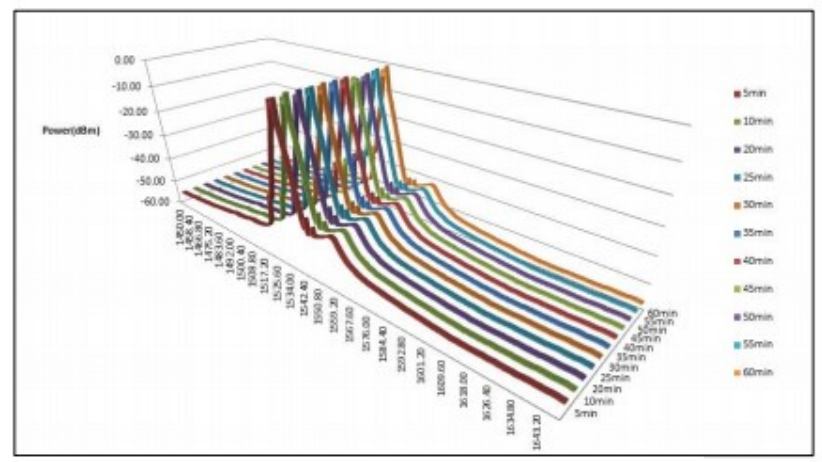

Fig. 10. The measured of OSA traces every $5 \min$ for $1 \mathrm{~h}$ for QSwitching.

As expected, the increment the pump power lead to increment of the pulse train or repetition rate and decrement the pulse width. As the input pump was raised from $200 \mathrm{~mW}$ to $600 \mathrm{~mW}$, the repetition rate expanded from $26.18 \mathrm{kHz}$ to $52.23 \mathrm{kHz}$ while the pulse width diminished starting from $0.2 \mu$ s to $0.1 \mu$ s. Fig. 11 show the pulse train from the oscilloscope for $600 \mathrm{~mA}$. Then, toward each pump energy and pulse repetition rate, no amplitude modulations in these pulse trains were observed and the Q-switched pulse output was stable. This demonstrates that there might have been no selfmode locking impact throughout the Q-switching operation. Lastly, the value of pulse spacing is $0.2 \mu \mathrm{s}$.

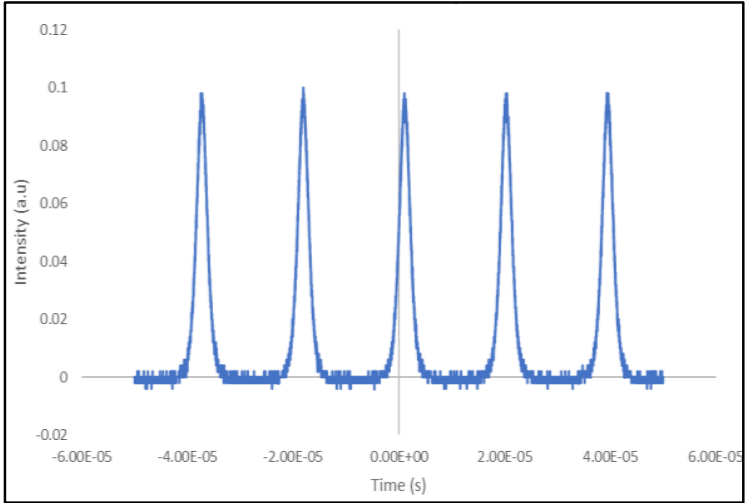

Fig. 11. Pulse train from the oscilloscope for $600 \mathrm{~mA}$

\section{Conclusions}

In summary, Q-switching of erbium-doped fiber laser (EDFL) is reported by using graphene layer as a saturable absorber. The graphene layer SA was sandwiched between two fiber ferrules to act as a passive Q-switch device. CW operation is achieved at $10 \mathrm{~mW}$, whereas stable self-starting Q-switching with a central wavelength of $1530 \mathrm{~nm}$ begins at $18 \mathrm{~mW}$. In this paper, at $35 \mathrm{~mW}$, the maximum pulse energy reaches at $2 \mu \mathrm{J}$ with pulse repetition rate of $1 \mathrm{MHz}$ and the narrowest pulse width is around $10 \mu \mathrm{s}$ is obtained. The stability of the pulse is verified from the radio-frequency (RF) spectrum with a measured signal-to-noise ratio (SNR) of $48 \mathrm{~dB}$. This work highlights the feasibility and simplicity of graphene layer as SA for femtosecond pulse generation that may have great potential in many applications such as supercontinuum generation.

We would like to thanks to Universiti Tun Hussein Onn Malaysia (UTHM) for providing GPPS Grant of (U753), (U757), RAGS Grant (R046) for sponsoring this work.

\section{References}

1. H. Ahmad, N. E. Ruslan, M. A. Ismail, S. A. Reduan, C. S. J. Lee, S. Sathiyan, S. Sivabalan, and S. W. Harun, Appl. Opt., vol. 55 (2016)

2. Zhao L M, Bartnik A C, Tai Q Q and Wise F W 2013 Opt. Lett. 381942

3. Kharenko D, Podivilov E, Apolonski A and Babin S 2012 Opt. Lett. 374104

4. Aguergaray C, Broderick N G R, Erkintalo M, Chen J S Y and Kruglov V 2012 Opt. Express 2010545

5. Okhotnikov O G, Jouhti T, Konttinen J, Karirinne S and Pessa M 2003 Opt. Lett. 28364

6. Yamashita S, Inoue Y, Maruyama S, Murakami Y, Yaguchi H, Jablonski M and Set S Y 2004 Opt. Lett. 291581 
7. H Ahmad, Julian M Semangun, Saaidal R Azzuhri, M Z Zulkifli, N A Awang and S W Harun, Laser Phys. 25 (2015)

8. H. H. Kuo and S. F. Hong, J Nanomater., vol. 14, (2014)

9. B. Xu, A. Martinez, S. Y. Set, C. S. Goh, and S. Yamashita, IEEE Photonics Technol. Lett., vol. 262 (2014)

10. J. Sotor, I. Pasternak, A. Krajewska, W. Strupinski, and G. Sobon, Optics Exp. vol. 23, no. 21, (2015).

11. J. Lee, J. Lee, J. Koo, and J. H. Lee, Appl. Opt., vol. 55, no. 2, (2016).

12. H. Ahmad, J.M. Semangun, S.R. Azzuhri, M.Z., Zulkifli, N.A. Awang and S.W. Harun, Laser Physics, 25 (4) (2015)

13. A. Hamzah, M.C. Paul, N. A. Awang, H. Ahmad, M. Pal, S. Das, M. A. Ismail, and S. W. Harun Optics and Laser Technology, vol. 47 (2013)

14. Z. Sun, T. Hasan, and A. C. Ferrari, Phys. E Lowdimensional Syst. Nanostructures, vol. 44, no. 6 (2012) 